\title{
The Diffusion of Chalcogen in $\mathrm{CdS}_{1-x} \mathrm{Se}_{x}$
}

\author{
By Masayoshi Nakano* and Kenzo Igaki**
}

\begin{abstract}
The diffusion coefficient of chalcogen in $\mathrm{CdS}_{1-x} \mathrm{Se}_{x}$ was measured for a specimen annealed under controlled chalcogen pressures using a reservoir containing molten $\mathbf{S}-\mathrm{Se}$ mixtures. The diffusion coefficient $D$ is independent of the composition $x$ in the range $0<x<0.5$ and proportional to the square root of the sum of partial pressures of $S_{2}(\mathrm{~g})$ and $\mathrm{Se}_{2}(\mathrm{~g})$. The estimated temperature dependence of $D$ near the stoichiometric composition is $D=2.2 \times 10^{-5} \exp \left(-4.2 \times 10^{-19} \mathrm{~J} / \mathrm{kT}\right)\left(\mathrm{m}^{2} / \mathrm{s}\right)$ $(1064 \mathrm{~K} \sim 1281 \mathrm{~K})$.
\end{abstract}

(Received August 26, 1981)

\section{Introduction}

The impurity diffusion of Se in CdS under the saturation sulfur vapor pressure was studied by Woodbury and Hall ${ }^{(1)}$ using radio isotope tracer. The interdiffusion coefficient in $\mathrm{CdS}_{1-x} \mathrm{Se}_{x}$ was measured by Handelman and Kaiser $^{(2)}$ and by Taylor et al. ${ }^{(3)}$ by means of color change with an optical microscope and fluorescence measurement, respectively. According to Woodbury and Hall, the penetration curve has two stages, that is, a slow diffusion stage in the high tracer concentration part and a fast stage in the low concentration part. The diffusion coefficient is found to be proportional to the square root of $S$ vapor pressure. But there has been no systematic investigation of the chalcogen diffusion in $\mathrm{CdS}_{1-x} \mathrm{Se}_{x}$. In the present paper, the diffusion coefficient has been studied on a sample annealed under the regulated chalcogen partial pressure using a reservoir containing molten S-Se mixtures.

\section{Experimental}

A specimen cleaved from a vapor grown CdS single crystal was annealed in a sealed quartz ampoule using a molten S-Se reservoir. This method was described in detail in a pre-

* Graduate School, Tohoku University, Sendai. Present address: ULVAC Corp., Hagisono, Chigasaki 253, Japan.

* Department of Materials Science, Faculty of Engineering, Tohoku University, Sendai 980, Japan. vious paper $^{(4)}$. The sample was quenched in water after annealing for $20 \mathrm{ks}-360 \mathrm{ks}$. It was sometimes sealed with $\mathrm{Ar}$ up to $70 \mathrm{kPa}$ at room temperature in order to diminish the loss of sample through vaporization. The composition $x$ in the sample $\left(\mathrm{CdS}_{1-x} \mathrm{Se}_{x}\right)$ was measured by an electron probe X-ray microanalyzer (EPMA). It was regulated by controlling the partial pressure ratio of $\mathrm{S}_{2}(\mathrm{~g})$ and $\mathrm{Se}_{2}(\mathrm{~g}),\left(P_{\mathrm{S}_{2}} / P_{\mathrm{Se}_{2}}\right)$. The following assumption was used on regulating the atom ratio of metal to chalcogen by the sum of partial pressures of $\mathrm{S}_{2}(\mathrm{~g})$ and $\mathrm{Se}_{2}(\mathrm{~g})$ under a fixed partial pressure ratio.

(1) The vapor pressures, $P_{\mathrm{S}_{2}}$ and $P_{\mathrm{Se}_{2}}$, over the specimen are proportional to the activities of $\mathrm{S}_{2}$ and $\mathrm{Se}_{2}$ in the molten $\mathrm{S}-\mathrm{Se}$ mixture reservoir, respectively. Partial pressures do not change at the vapor temperature between $1050 \mathrm{~K}$ and $1300 \mathrm{~K}$ at a fixed composition and temperature of reservoir.

(2) Molten S-Se mixture is an ideal solution. As a reference condition for changing temperature and composition, the reservoir temperature $T_{\mathrm{R}}=723 \mathrm{~K}$ and the atomic fraction of $\mathrm{S}$ in the reservoir $N_{\mathrm{S}}=0.650$ were adopted. The value of $\left(P_{\mathrm{S}_{2}} / P_{\mathrm{Se}_{2}}\right)$ was 3.6 in this condition, as described in Fig. 10 in the previous report ${ }^{(4)}$. Then the following eq. (1) was derived:

$$
\begin{aligned}
& (0.650 / 0.350)\left(P_{\mathrm{S}_{2} 723}^{\circ} / P_{\mathrm{Se}_{2} 723}^{\circ}\right) \\
& \quad=\left\{N_{\mathrm{S}} /\left(1-N_{\mathrm{S}}\right)\right\}\left(P_{\mathrm{S}_{2} T_{\mathrm{R}}}^{\circ} / P_{\mathrm{Se}_{2} T_{\mathrm{R}}}^{\circ}\right),
\end{aligned}
$$

where $P_{\mathrm{S}_{2} T_{\mathrm{R}}}^{\circ}$ and $P_{\mathrm{Se}_{2} T_{\mathrm{R}}}^{\circ}$ are the equilibrium pressures of pure $\mathrm{S}$ and Se at $T_{\mathrm{R}}$, respectively. At first $N_{\mathrm{S}}$ and $T_{\mathrm{R}}$ were determined with eq. (1). In order to estimate $P_{\mathrm{S}_{2}}$ and $P_{\mathrm{Se}_{2}}$, the 
following equations were used:

$$
\begin{aligned}
& P_{\mathrm{S}_{2}}=0.26 N_{\mathrm{S}} P_{\mathrm{S}_{2} T_{\mathrm{R}}}^{\circ}, \\
& P_{\mathrm{Se}_{2}}=7.3\left(1-N_{\mathrm{S}}\right) P_{\mathrm{Se}_{2} T_{\mathrm{R}}}^{\circ} .
\end{aligned}
$$

Here the constants were determined using the relation between $\left(P_{\mathrm{S}_{2}} / P_{\mathrm{Se}_{2}}\right)$ and $N_{\mathrm{S}}$ shown in Fig. 10 in the previous report ${ }^{(4)}$, the equilibrium constant of the reaction between $\mathrm{S}_{2}(\mathrm{~g})$, $\mathrm{Se}_{2}(\mathrm{~g})$ and $\mathrm{SSe}(\mathrm{g})^{(5)}$, and the relation between chalcogen total pressure of the S-Se system and $N_{\mathrm{S}}$ reported by Devyatykh et al. ${ }^{(6)}$. This estimation is rough, but the validity will be checked experimentally by the constant surface composition of crystals.

\section{Calculation Method of Diffusion Coefficient}

Because the chemical potential at the surface of the sample is constant in the experimental condition of this study, the Fick's second law,

$$
\partial c / \partial t=D \partial^{2} x / \partial y^{2}
$$

is solved in the initial condition, $x=0$ at $y>0$ and $t=0$, and in the boundary condition, $x=x_{0}$ at $y=0$ and $t>0$, where $D$ is the diffusion coefficient, $x$ is the concentration at the depth $y, x_{0}$ is the surface concentration and $t$ is the diffusion time. The solution is expressed by the following equation ${ }^{(7)}$ :

and

$$
x=x_{0} \operatorname{erfc}\{y /(2 \sqrt{D t})\},
$$

$$
\operatorname{erfc}(z)=1-\operatorname{erf}(z)=1-(2 / \sqrt{\pi}) \int_{0}^{z} e^{-\eta^{2}} \mathrm{~d} \eta .
$$

Equation (5) is derived on the assumption that $D$ is independent of $x$, and is used to be independent of the concentration. If the experimental result satisfies eq. (5), $D$ is known to be independent of the concentration.

The ordinate of a normal probability paper is graduated with the cumulative normal probability function,

$$
\mathrm{F}(z)=(1 / \sqrt{2 \pi}) \int_{-\infty}^{z} e^{-\tau^{2} / 2} \mathrm{~d} \tau .
$$

Replacing $\tau$ by $\sqrt{2 \eta}$, the following equation is derived:

$$
\operatorname{erf}(z / \sqrt{2})=2 \mathrm{~F}(z)-1
$$

Equation (5) gives the following expression:

$$
\operatorname{erf}\{y / 2 \sqrt{D t}\}=1-\left(x / x_{0}\right) .
$$

The following equation is derived from eqs. (7) and (8).

$$
\mathrm{F}(y / \sqrt{2 D t})=1-\left(x / 2 x_{0}\right) .
$$

When $1-\left(x / 2 x_{0}\right)$ is plotted against $y$ on a normal probability paper, the plot gives a straight line and its slope is equal to $(2 D t)^{-1 / 2}$, if the experimental data satisfy eq. (5). As a first step the linearity was confirmed with the plot on a normal probability paper, and then the slope of the line was calculated using the numerical table between $z$ and $\mathrm{F}^{-1}(z)$ by the method of least squares. The diffusion coefficient was determined from the slope.

\section{Results and Discussion}

In Fig. 1 is shown an example of the penetration curve at the sample temperature $T_{\mathrm{S}}=$ $1273 \mathrm{~K}$, the reservoir temperature $T_{\mathrm{R}}=723 \mathrm{~K}$, the atomic fraction of $\mathrm{S}$ in reservoir $N_{\mathrm{S}}=0.650$ and the diffusion time $t=86.4 \mathrm{ks}$. The surface concentration $x_{0}$ was obtained to be $x_{0}=$ 0.192 by extrapolating the profile to the surface $(y=0)$. In Fig. 2 is shown the relation between $\mathrm{F}^{-1}\left\{1-\left(x / 2 x_{0}\right)\right\}$ and the distance from the surface $y$. This plot gives a straight line. The value of $D$ was obtained from the slope of the straight line to be $6.2 \times 10^{-15} \mathrm{~m}^{2} / \mathrm{s}$. The solid line in Fig. 1 is a calculated line with

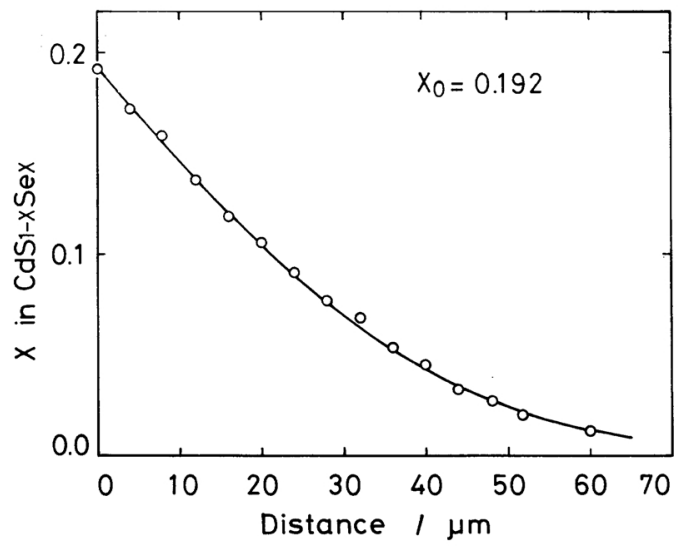

Fig. 1 An example of penetration curve. $\left[T_{\mathrm{S}}=1273\right.$ $\left.\mathrm{K}, T_{\mathrm{R}}=723 \mathrm{~K}, N_{\mathrm{S}}=0.650, t=86.4 \mathrm{ks}(24 \mathrm{~h})\right]$. 


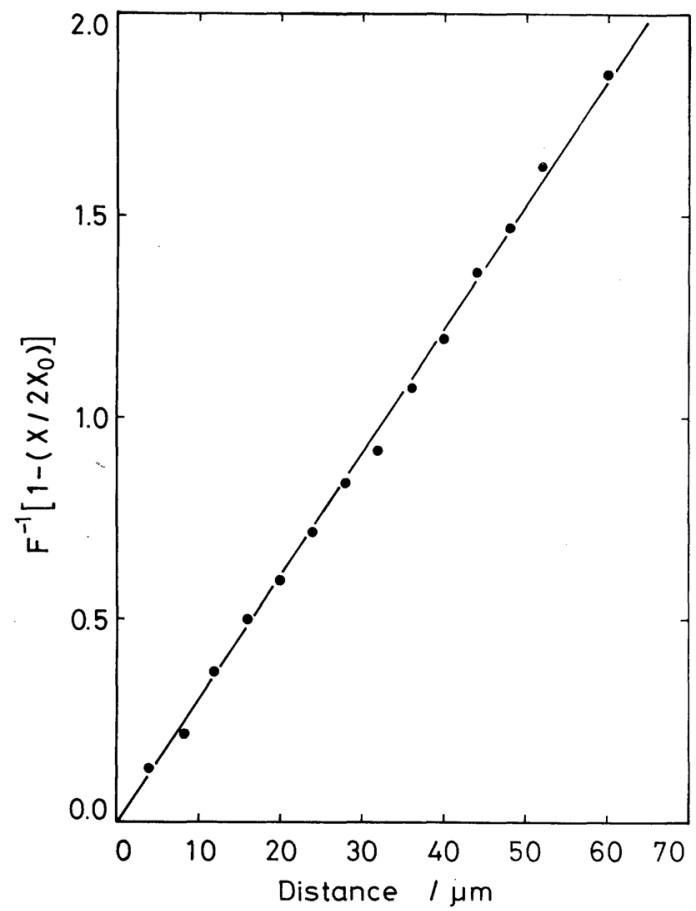

Fig. 2 Normal probability plot of the values in

Fig. 1.

this value in eq. (5). The normal probability paper plots give straight lines in several runs in the conditions, $0<x_{0}<0.5, T_{\mathrm{S}}=1273 \mathrm{~K}$, $1173 \mathrm{~K}$ or $1073 \mathrm{~K}$ and $T_{\mathrm{R}}=723 \mathrm{~K}$ or $637 \mathrm{~K}$, and so it is clarified that the diffusion coefficient is independent of composition in the range $0<$ $x<0.5$. In the surface composition range $0.5 \leqq$ $x_{0}<1$, the vaporization rate of the sample is too large to prove the linearity of the plot.

The surface compositions of annealed samples at $T_{\mathrm{S}}=1273 \mathrm{~K}$ and $1173 \mathrm{~K}$ are plotted against $\left(P_{\mathrm{S}_{2}}+P_{\mathrm{Se}_{2}}\right)$ at constant $\left(P_{\mathrm{S}_{2}} / P_{\mathrm{Se}_{2}}\right)$ in Fig. 3. Experimental values of $x_{0}$ are $0.21 \pm$ 0.02 in the pressure range, $0.5 \mathrm{kPa}<\left(P_{\mathrm{S}_{2}}+\right.$ $P_{\mathrm{Se}_{2}}$ ) $<50 \mathrm{kPa}$ (where $0.3<N_{\mathrm{S}}<0.75$ and 563 $\mathrm{K}<T_{\mathrm{R}}<773 \mathrm{~K}$ ). It can be said that the estimation of the pressures using the molten S-Se reservoir is reasonable in a limited range because the composition of $\mathrm{CdS}_{1-x} \mathrm{Se}_{x}$ is regulated by $\left(P_{\mathrm{S}_{2}} / P_{\mathrm{Se}_{2}}\right)^{(2)(4)}$. In the range $\left(P_{\mathrm{S}_{2}}+\right.$ $\left.P_{\mathrm{Se}_{2}}\right)>50 \mathrm{kPa}$, however, $x_{0}$ tends to decrease steeply, and the estimation of the pressures is not so good. The value of $\left(P_{\mathrm{S}_{2}} / P_{\mathrm{Se}_{2}}\right)$ is considered to deviate from the estimated one to a higher one. The diffusion coefficient $D$ is plotted

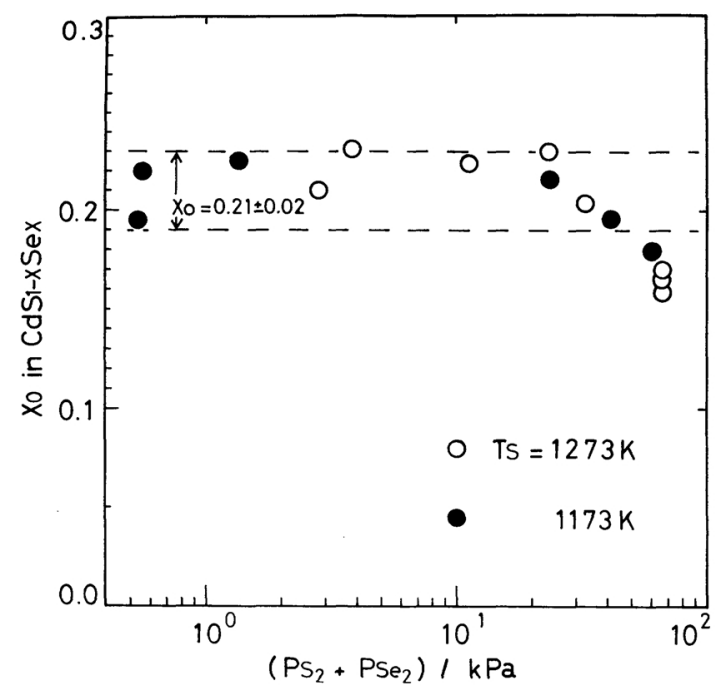

Fig. 3 Surface composition as a function of $\left(P_{\mathbf{S}_{2}}+\right.$ $\left.P_{\mathrm{Se}_{2}}\right)$ at $P_{\mathrm{S}_{2}} / P_{\mathrm{Se}_{2}} \doteqdot 3.6$. The broken lines show the composition range $x_{0}=0.21 \pm 0.02$.

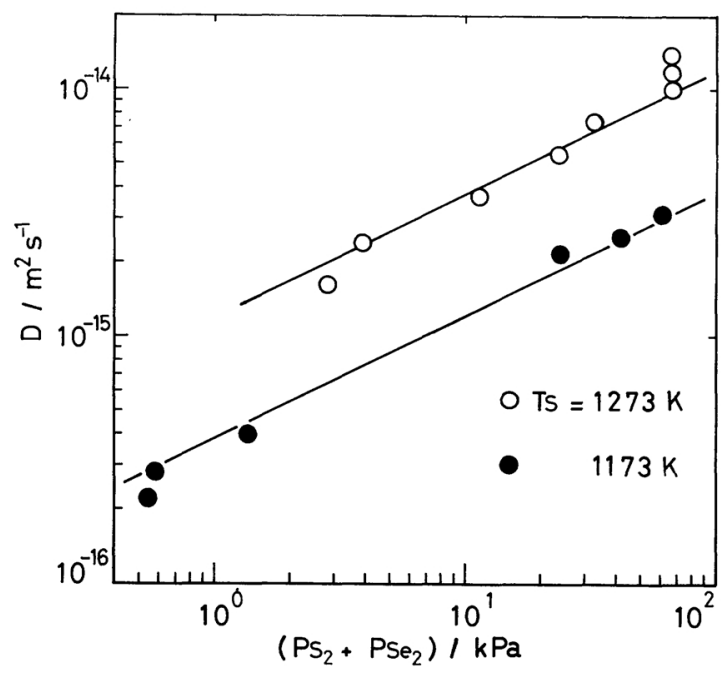

Fig. 4 Diffusion coefficient as a function of $\left(P_{\mathrm{S}_{2}}+\right.$ $\left.P_{\mathrm{Se}_{2}}\right)$ on the samples in Fig. 3. The solid lines are proportional to $\left(P_{\mathrm{S}_{2}}+P_{\mathrm{Se}_{2}}\right)^{1 / 2}$.

against $\left(P_{\mathrm{S}_{2}}+P_{\mathrm{Se}_{2}}\right)$ in Fig. 4 on the samples in Fig. 3. The data are nearly on the solid lines proportional to $\left(P_{\mathrm{S}_{2}}+P_{\mathrm{Se}_{2}}\right)^{1 / 2}$. Thus $D$ is known to be nearly proportional to $\left(P_{\mathrm{S}_{2}}+P_{\mathrm{Se}_{2}}\right)^{1 / 2}$ at constant $x$ and $\left(P_{\mathrm{S}_{2}} / P_{\mathrm{Se}_{2}}\right)$.

Subsequently $D$ was measured changing $T_{\mathrm{S}}$ from $1064 \mathrm{~K}$ to $1281 \mathrm{~K}$ at $N_{\mathrm{S}}=0.650$ and $T_{\mathrm{R}}=723 \mathrm{~K}(\mathrm{O})$ or $673 \mathrm{~K}(\square)$. The surface composition of the samples are $x_{0}=0.20 \pm$ 
0.02. $D$ is plotted against the reciprocal absolute samples temperature in Fig. 5. The upper solid line can represent the obtained data and is given by the following equation determined by the method of least squares on the values for $T_{\mathrm{R}}=723 \mathrm{~K}$ :

$$
\begin{gathered}
D=3.8 \times 10^{-9} \exp \left(-2.4 \times 10^{-19} \mathrm{~J} / k T\right) \\
\left(\mathrm{m}^{2} / \mathrm{s}\right), \\
(1064 \mathrm{~K} \sim 1281 \mathrm{~K})
\end{gathered}
$$

where $k$ is Boltzmann's constant.

The values represented by open symbols $\bigcirc$ and $\square$ are calculated for the cases corresponding to the minimum of the total pressure using the relation $D \propto\left(P_{\mathrm{S}_{2}}+P_{\mathrm{Se}_{2}}\right)^{1 / 2}$. This plot shows the temperature dependence of $D$ near the stoichiometric composition. The lower solid line is given by the following equation:

$$
\begin{array}{r}
D=2.2 \times 10^{-5} \exp \left(-4.2 \times 10^{-19} \mathrm{~J} / k T\right) \\
\left(\mathrm{m}^{2} / \mathrm{s}\right) .
\end{array}
$$

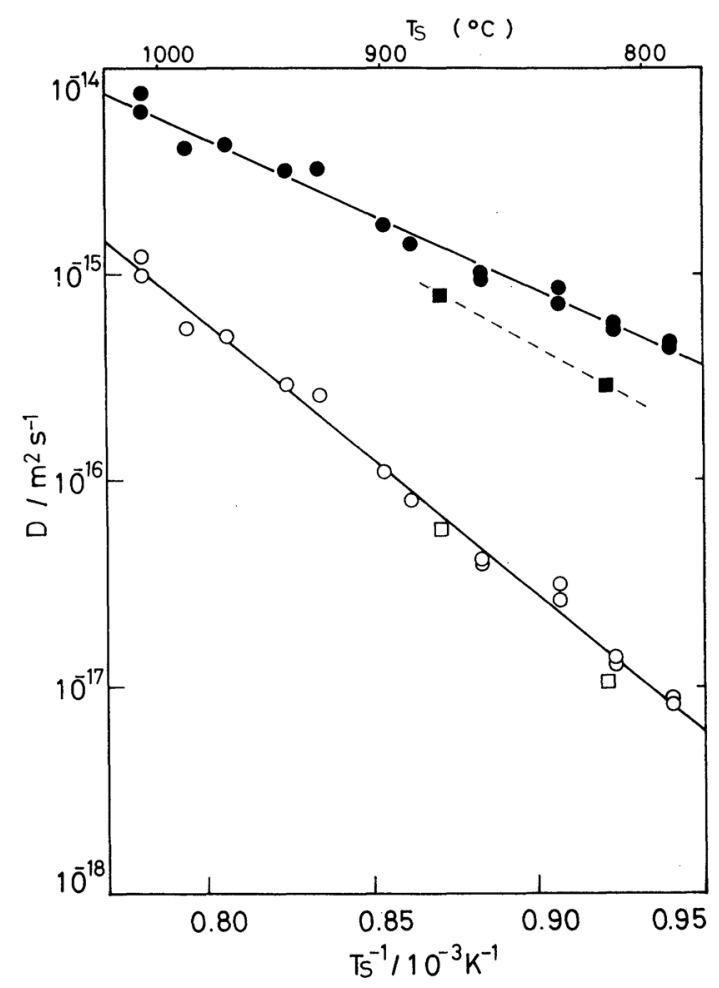

Fig. 5 Diffusion coefficient as a function of the reciprocal absolute sample temperature. $\bigcirc: T_{\mathrm{R}}=$ $723 \mathrm{~K}, \square: T_{\mathrm{R}}=673 \mathrm{~K}\left[N_{\mathrm{S}}=0.650\right]$. Open symbols $\bigcirc$ and $\square$ are the calculated values for the minimum of the total pressure.
The dissociation constant of $\mathrm{CdS}_{1-x} \mathrm{Se}_{x}$ is estimated by the following equation which is used in calculating the pressure corresponding to the minimum of the total pressure:

$$
\begin{aligned}
K_{\mathrm{CdS}_{1-x} \mathrm{Se}_{x}} & =P_{\mathrm{Cd}}^{2} \times\left(P_{\mathrm{S}_{2}}+P_{\mathrm{Se}_{2}}\right) \\
& =a_{\mathrm{CdS}}^{2} K_{\mathrm{CdS}}+a_{\mathrm{CdSe}}^{2} K_{\mathrm{CdSe}},
\end{aligned}
$$

where $K_{\mathrm{CdS}}$ and $K_{\mathrm{CdSe}}$ are dissociation constants of the component compounds $\mathrm{CdS}^{(8)}$ and $\mathrm{CdSe}^{(9)}$, respectively, and $a_{\mathrm{CdS}}$ and $a_{\mathrm{CdSe}}$ are activities of $\mathrm{CdS}$ and CdSe in ternary compounds $\mathrm{CdS}_{1-x} \mathrm{Se}_{x}^{(4)}$, respectively.

The relation, $D \propto\left(P_{\mathrm{S}_{2}}+P_{\mathrm{Se}_{2}}\right)^{1 / 2}$, is obtained for constant $\left(P_{\mathrm{S}_{2}} / P_{\mathrm{Se}_{2}}\right)$ as shown in Fig. 4. The diffusion of chalcogen atoms under high chalcogen pressure is suggested to be due to neutral interstitial atoms for $\mathrm{CdS}$ and $\mathrm{CdSe}^{(10)}$. This suggestion is derived from the experimental results that the diffusion coefficient of chalcogen is proportional to the square root of chalcogen vapor pressure and that no change of diffusion coefficient is observed for any doping donor impurity (for example In). On the other hand, under a low chalcogen pressure, that is, under a high $\mathrm{Cd}$ vapor pressure, the diffusion of chalcogen is suggested to be due to chalcogen vacancies as well as neutral interstitial atoms.

The relation observed, $D \propto\left(P_{\mathrm{S}_{2}}+P_{\mathrm{Se}_{2}}\right)^{1 / 2}$, can be interpreted well by assuming the diffusion mechanism of chalcogen as neutral interstitial atoms. Namely, Se neutral interstitial atoms diffuse into CdS from the surface, and replace $S$ atoms at $S$ sites. Then $S$ neutral interstitial atoms diffuse out to the surface. These two diffusion processes are affected by $P_{\mathrm{Se}_{2}}$ and $P_{\mathrm{S}_{2}}$, respectively.

\section{Summary}

The diffusion coefficient of chalcogen in $\mathrm{CdS}_{1-x} \mathrm{Se}_{x}$ is studied on annealed samples under the controlled chalcogen partial pressures by using a molten $\mathrm{S}-\mathrm{Se}$ reservoir. The diffusion coefficient $D$ is independent of composition $x$ in the range $0<x<0.5$ at the sample temperature $T_{\mathrm{S}}=1173 \mathrm{~K}$ and $1273 \mathrm{~K}$. The temperature dependence of $D$ near the stoichiometric composition is estimated in the temperature range between $1064 \mathrm{~K}$ and 
$1281 \mathrm{~K}$. The diffusion of chalcogen atoms is deduced to be due to neutral interstitial atoms, from the relation $D \propto\left(P_{\mathrm{S}_{2}}+P_{\mathrm{Se}_{2}}\right)^{1 / 2}$.

\section{REFERENCES}

(1) H. H. Woodbury and R. B. Hall: Phys. Rev., 157 (1967), 641.

(2) E. T. Handelman and W. Kaiser: J. Appl. Phys., 35 (1964), 3519.

(3) H. F. Taylor, V. N. Smiley, W. E. Martin and S. S. Pawka: Phys. Rev., B5 (1972), 1467.

(4) M. Nakano and K. Igaki: Trans. JIM, 21 (1980), 739.
(5) M. Nakano and K. Igaki: Trans. JIM, 21 (1980), 734.

(6) G. G. Devyatykh, A. I. Odnosevtsev and V. A. Umilin: Zhr. Neorg. Khim., 7 (1962), 1928.

(7) J. Crank: The Mathematics of Diffusion, 2nd ed., Clarendon Press, Oxford (1975), p. 20.

(8) P. Goldfinger and N. Jeunehomme: Trans. Faraday Soc., 59 (1963), 2851.

(9) K. Igaki and M. Nakano: Trans. JIM, 20 (1979), 597.

(10) D. A. Stevenson: Atomic Diffusion in Semiconductors, Ed. by D. Shaw, Plenum Press, London and New York, (1973), p. 431. 